\title{
Spontaneous Access and Analogical Incubation Effects
}

\author{
Bo T. Christensen \\ University of Aarhus \\ Christian D. Schunn \\ University of Pittsburgh
}

\begin{abstract}
Incubation often plays a role in creative problem solving. Theories of analogical problem solving and Opportunistic Assimilation (OA) theory (Seifert, Meyer, Davidson, Patalano, \& Yaniv, 1995) of incubation in creative problem solving were tested. OA theory predicts that a previously encoded unsolved problem will be spontaneously accessed and mapped by a later encounter with an analogous problem-with-solution. This study tested the OA predictions on insight problems and found spontaneous access effects for uninformed participants. Spontaneous access was compared to a baseline derived from the same participant's typical problem-solving behavior following distracter tasks. Following access of the analogous cue, participants increased their performance, demonstrating incubation effects. These results support the theory that at least some incubation effects are caused by previously unsolved problems being solved on later chance encounters with relevant information in the environment.
\end{abstract}

Since Gick and Holyoak $(1980,1983)$ conducted their classic studies of Duncker's (1945) radiation problem, the standard way of studying analogical problem solving has consisted of first letting participants encode a problem-with-solution (source), and then afterward presenting the participants with an analogous problem to solve (target). This task structure is mirrored in prevailing models of reasoning by analogy and similarity such as MAC/FAC (Forbus, Gentner, \& Law, 1994), LISA (Hummel \& Holyoak, 1997), and ARCS (Thagard, Holyoak, Nelson, \& Gochfeld, 1990). The canonical model divides analogical problem-solving into at least three phases: First, the source is encoded; second, a target induces access of a source relevant to the solution of the target; and third, the source is mapped unto the target to draw a solution (e.g., Anolli, Antonietti, Crisafulli, \& Cantoia, 2001).

However, experiment V of Gick and Holyoak (1980, experiment V) also included a variation of this analogical problem solving ordering. The experimental variation consisted of changing the order of the source and target, by first allowing the participants time to work on the radiation problem (target), followed by reading and recall of an analogous story (source-the Attack-Dispersion story), again followed by work on the radiation problem. This "target source" condition was compared to the standard way of doing analogical problem solving (i.e., source before target), and to a control receiving no analogous story, but with incubation time away from the problem. There was no indication that the target-source condition increased performance over the source-target condition, but receiving the analogous story did improve performance over the incubation control condition. This variation of the standard analogical problem solving paradigm has only been used a few times in the analogical problem solving literature.

Another study using this "reverse" (i.e., target-source) analogical problem-solving design was by Keane (1985). That study tested whether presenting

The authors would like to thank Kwangsu Cho, Anthony Harrison, Xiaohui Kong, and Mark McGregor for helpful comments on an earlier draft. This work was supported by Grant N00014-01-1-0321 from the U.S. Office of Naval Research to the second author.

Correspondence and requests for reprints should be sent to Bo T. Christensen, Department of Psychology, University of Aarhus, Jens Chr. Skous Vej 4, 800 Aarhus C, Denmark. E-mail: botc@psy.au.dk 


\section{B. T. Christensen and C. D. Schunn}

merely the solutions to analogous problems was enough to improve performance over an incubation control group, again using the radiation problem, but this time using distracter tasks. Participants were instructed that the solutions might be useful. This hypothesis was supported.

Although the dominant theories of analogical access would not predict any difference for these two different directions of analogical retrieval, it is especially interesting for studies of creativity to examine this "reverse" direction further. For creative problems, where the solution can be surprising and may restructure the entire problem representation (Dominowski \& Dallob, 1995; Kaplan \& Simon, 1990), this ordering may be important because a restructured problem space may analogically trigger access to different source analogs. This study was a careful time-course analysis of "reverse" analogical problem solving using video recordings of the problem-solving process. By using this nonstandard "reverse" analogical problem-solving design we were able to code for impasse, incubation, spontaneous access, and mapping in the same design.

In the sections that follow, we will briefly review the relevant pieces of the analogical transfer literature and then the incubation literature.

\section{Analogical Transfer: Access, Retrieval, and Noticing}

A number of factors have been found to influence spontaneous analogical access. Access is improved when the source is presented as a problem rather than as declarative knowledge (Adams, Kasserman, Yearwood, \& Perfetto, 1988; Lockhart, Lamon, \& Gick, 1988), if explicitness of the cross-domain relational similarity is increased (Clement, Mawby, \& Giles, 1994), or if experimental conditions stress a rich encoding and structural information at retrieval (Dunbar, 2001). Furthermore, it has been found that the presence of salient superficial similarity increases spontaneous access (e.g., Holyoak \& Koh, 1987; Gentner, Rattermann, \& Forbus, 1993).

A large number of studies have found that providing participants with an analogous source prior to showing the target rarely leads to significant access (or mapping) of the source, unless explicit instructions are provided to make use of previous information (i.e., participants are informed of the potential relevance of previous problems during the instructions), or if the source is very similar to the target (see, e.g., Anolli et al., 2001, for a brief review). Anolli et al. (2001) argued that these findings suggested that analogical access is not a spontaneous process. However, as Ross, Ryan, and Tenpenny (1989) noted, studies have not shown that people never spontaneously access relevant information, but only that, in cases where they are expected to do so, they often do not. Trying to measure spontaneous access on uninformed participants is a methodological problem that previous studies have struggled with. Typically either informed participants are used in a paradigm where the participants are asked to write down any previous problems they access (e.g., Gentner et al., 1993), or performance measures are used (i.e., correct or incorrect solution), which is a less optimal measure of access because some participants may have accessed the source without being able to map between source and target (e.g., Gick \& Holyoak, 1980). An exception was Ross (1987), who used correct-incorrect performance to measure mapping, but used a different score (whether the participant appeared to be making use of a source formula on the answer sheet) to measure access. This measure enables a separation of access and mapping in the same design, but still runs the risk of underestimating access. For example, participants may access a source without making use of it on the answer sheet, because they do not consider it relevant.

As Ross et al. (1989) argued, an important aspect of measuring spontaneous access in a within-subject design is that the participant should not be aware of the relevance of the source analogues (i.e., be informed). When measuring spontaneous access in a within-subject design it is thus necessary for the researcher to demonstrate that participants did not become aware of the relevance (i.e., catch on, thus becoming informed) after they had accessed relevant sources. This result led Ross et al. (1989) to conclude that, to avoid having to deal with an unknown mixture of informed and uninformed cases, access issues need to be studied with informed participants.

Efforts were made in this study to design a novel way of measuring spontaneous access on uninformed participants, while avoiding the above pitfalls, by separating access and mapping measures in a within-subject design while controlling for whether the participants had caught on. 
A note on terminology: Ross (1989) has made a distinction between access, noticing, and retrieval of a source. Access involves both noticing and retrieving a source. Where the noticing involves recognizing or selecting a particular earlier example, retrieval concerns the actual remembrance of this earlier example. Further, the analogical access literature have used terms like spontaneous (e.g., Holyoak \& Koh, 1987) and automatic (e.g., Anolli et al., 2001) for experimental conditions where the participant is uninformed about relevance of the source information. In this study, the term immediate access refers to access of the relevant unsolved target problem immediately following presentation of an analogous source problem. The term spontaneous immediate access is used to refer to immediate access where the participants are also uninformed about the relevance of the source information for solving the problem, and where they have not yet caught on to this fact. The term automatic is not used in order to avoid suggesting a constant law or high frequency of access because this suggestion seems unwarranted given the hard time researchers have had finding evidence for this phenomenon.

\section{Incubation as Reversed Analogical Transfer}

The "reversed" analogical problem solving design (i.e., target source) is identical with incubation effect study designs, and thus allows for testing of incubation effect hypotheses. The incubation effect refers to the phenomenon that spending time away from a problem may be beneficial to performance, compared to continuous problem solving. Incubation starts off with an individual who, having reached an impasse on a problem, sets the problem aside. During incubation, no conscious problem solving appears to be going on, and individuals go about their other unrelated business. At some point the individual will return to problem solving, often described as occurring suddenly and surprisingly, in a flash of "insight," where the solution is readily evident. Experimental studies of this phenomenon have received mixed results. An early review (Olton, 1979) questioned the existence of the incubation phenomenon, but a more recent review (Dodds, Ward, \& Smith, in press) argued that recent studies show more promise.

Incubation theories can be divided into two groups: Autonomous incubation refers to theories hypothesiz- ing that the effect is due to some phenomenon relying only on the passage of time, whereas interactive incubation theories require that new relevant information must be available in the environment (Dorfman, Shames, \& Kihlstrom, 1996). Autonomous theories of incubation have, for example, attributed the phenomenon to unconscious idea generation (e.g., Campbell, 1960), selective forgetting (Simon, 1966), and forgetting of fixating elements (Smith, 1995; Smith \& Blankenship, 1991). However, the interactive theory of Opportunistic Assimilation (OA; Seifert et al., 1995; Patalano \& Seifert, 1994) tries to explain incubation effects with chance (e.g., analogical) cuing from the environment. The theory states that reaching an impasse on a creative problem will encode "failure indices" in memory, which will be activated through automatic spreading of activation by standard perception and comprehension processes if the participant later encounters an analogous solution or solution element in the environment, thus bringing the previously unsolved problem back into awareness for new solution attempts. These failure indices constitute a form of predictive encoding that allows the individual to recognize later opportunities to achieve pending goals (Patalano $\&$ Seifert, 1997; Seifert, 1994), much like it occurs in everyday planning. In this way, the theory ties in with the Zeigarnik (1927) effect in that it hypothesizes that memory for unsolved problems is better than for solved problems, and with Louis Pasteur's notion that "chance favors the prepared mind" (e.g., Posner, 1973). OA thus predicts that seeing relevant information in the environment will trigger spontaneous access to previously unsolved analogous problems. Furthermore, this access is predicted to increase performance on the previously unsolved problem. Both of these hypotheses can be tested using the "reverse" analogical problem solving paradigm.

On testing the importance of impasses in OA theory, Seifert et al. (1995) found that only when participants had previously reached an impasse in answering difficult factual questions, and when they were later followed by incidental relevant information, did participants improve performance. Patalano and Seifert (1994) investigated the memorability of solved and unsolved problems following impasse, and found this effect depended on set size. Only when impasses are infrequent (i.e., when set size is small), are unsolved problems more available in memory than solved problems. 


\section{B. T. Christensen and C. D. Schunn}

Few studies have tested for interactive incubation effects, and with mixed results. An early study by Dreistadt (1969) showed that the presentation of visual analogical cues during incubation on insight problems had a significant effect on performance when compared to participants working continuously on the problem. However, Olton and Johnson (1976) failed to replicate this result, and Browne and Cruse (1988) using one of Dreistadt's insight problems found incubation effects for the experimental group receiving cues in only one of their two experiments. Dominowski and Jenrick (1972) found incubation effects for providing verbal cues on the hat-rack problem. Remote Associates Test (RAT) items (Mednick, 1962) have been used in several studies; Mednick et al. (1964, exp II) found that participants performed better on items where they were primed with cues during incubation than when they were not. Using a variation of RAT items, Dorfman (1990) found mixed results depending on whether the incubation task consisted of working on other similar problems (yielding an interactive incubation effect) or working on an unrelated arithmetic task (yielding an autonomous incubation effect). In a large study, Dodds, Smith, and Ward (2002) tested whether the presentation of associates of the answer would automatically lead to performance increases following initial solution attempts on RAT items, but no significant effect was found. It should be noted that, in all RAT studies, associates were used as cues, which is not the same as analogues.

In conclusion, studies testing for interactive incubation effects have yielded mixed results when using visual analogies for insight problems and RAT items.
However, as noted earlier, two studies have found incubation effects when providing analogous stories (Gick \& Holyoak, 1980, exp V) or solutions to analogous problems (Keane, 1985) during incubation. But both these studies suffer from shortcomings. Gick and Holyoak (1980, exp V) did not provide distracter tasks (only the Attack-Dispersion story was read during incubation). Adding distracter tasks could potentially have influenced spontaneous transfer, as indicated by their experiment IV, and possibly reduced spontaneous transfer to insignificant amounts given the small sample size used (10 participants in the control group, and 20 participants in the incubation condition). Keane (1985) did provide distracter tasks, but he also instructed participants that the stories they read might be relevant in solving the problem, thereby informing participants of the relevance of the cue. In summary, it has yet to be established what (if any) kinds of creative problems and cues will show reliable interactive incubation effects for uninformed participants. Moreover, none of these reverse analogy studies (nor in fact the majority of traditional analogy studies) have looked at the process of spontaneous access and success of analogical transfer in the same design. By using video in a careful time-course analysis this study measured impasse, incubation, analogical access, and analogical mapping separately in the same design. The study tested whether encountering analogous solutions to previously unsolved problems would lead to spontaneous immediate access of the unsolved problem, and performance increase through analogical mapping on insight problems (see Figure 1). Further, it was tested whether initial encoding time of the problem or incubation time predicted immediate retrieval of the un-

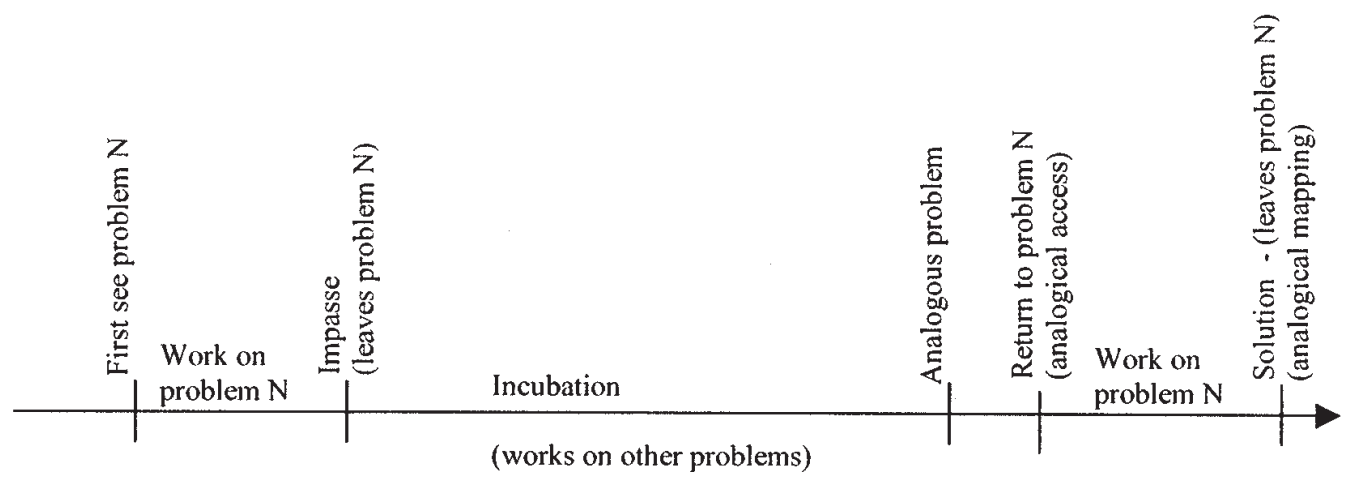

Figure 1. Problem-solving timeline on individual problems. 
solved problem on seeing the relevant analogue. Finally, categories that are typically not measurable in standard analogical problem-solving designs were examined; in particular we examined problems that are immediately accessed, but where no performance increase is found, and problems that are not immediately accessed, but where performance is nonetheless increased once the participant returns to the problem. These categories are typically not measurable because they require a separation of access and mapping measures in the same design.

In sum, we examined whether analogous cues do produce immediate access to related unsolved problems, and then whether access produce successful analogical mappings (thereby accounting for at least one source of incubation effects). It is possible that analogous cues do not produce immediate access, but instead produce analogical mappings only when people happen to return to the unsolved problem later. For cases with low surface similarity, this result could be expected from the frequent failures to find spontaneous analogical access with low surface similarity. It is also possible that analogous cues do produce immediate access of related unsolved problems, but that analogical mappings are not successfully made with high frequency (in which case OA is a poor account of incubation effects).

\section{Methods}

\section{Participants}

Forty undergraduates (12 female) from the University of Pittsburgh participant pool participated for course credits.

\section{Materials}

Participants worked on eight insight problems adapted from the literature (see Appendix A). Each problem was presented on a separate page. Eight analogous insight problems were constructed as cues (analogous cues), along with four unrelated insight problems (distracter tasks). These were presented as rating tasks, where the participants had to rate the problem for difficulty on a scale from 1 to 5 . These rating tasks were constructed so the problem was presented on the front, and the solution to the problem and the rating scale on the back of a sheet of paper. Rating tasks and problem order were varied across participants. Each participant received four analogous and four distracter rating tasks.

These experimental materials were constructed to minimize superficial similarity between source and target as much as possible. However, in designing the materials we found that the distinction between superficial similarity and structural similarity is somewhat subjective and may be a continuum rather than a dichotomy. We found that it is always possible for a motivated person to find some superficial similarity between problems that are supposed to contain "only" structural similarity. For example, in the present materials, the analogue we constructed to the Tennis Tournament problem was a Viking battle where Vikings fight each other to go to Valhalla (see Appendix A). This analogue was constructed to minimize story line similarity, leaving mainly structural similarity. However, some of the concepts used are somewhat interchangeable, although different words are applied; for example, the words match (as in tennis match) and fight (in the Viking story) basically point to the same underlying construct even though different words are applied. In some cases, these words can be used interchangeably, as in boxing match and boxing fight. Does this correspondence mean that the presence of match in the tennis story and the presence of fight in the Viking story constitute structural or superficial similarity? We suggest it has elements of both; the match-fight construct serves to secure a structurally relevant mapping, but insofar as the match-fight concepts are interchangeable (in a given problem context), they can also be characterized as somewhat superficially similar. To take another example from the Viking story, the words tournament and battle are again used as analogous concepts. In this case, however, the tournament (in the context of a tennis match) has additional constraints attached to it, compared to the "battle" concept. Tournaments involve a strict hierarchical structure, where the winner from one match moves to the next "level" in the tournament. This strict hierarchy is not an essential part of the "battle" concept, and thus this constraint had to be added to the Viking story to make the analogy a tight one. Thus, in this case, it has to be inferred by the problem solver that battle is analogous to tournament if this constraint is added. In this case, we argue the similarity between battle and tournament is more structural than superficial. 


\section{B. T. Christensen and C. D. Schunn}

Our materials appear to sit somewhere in the middle of the continuum between superficial and structural similarity. However, even though some amount of superficial similarity may be present in our materials, this does not make our results uninteresting to the "analogical access" literature. As Hammond, Seifert, and Gray (1991) argued, memory serves a function, and theories and experiments on memory should include (rather than exclude) those sets of useful features that are already part of the tasks that memory serves. Superficial similarity is one such feature. Furthermore, as briefly reviewed in the introduction, interactive incubation studies have had problems finding reliable results, which would make any positive incubation effect interesting - regardless of whether it is triggered in part by superficial similarity. Finally, problem-cue pairs were designed to minimize superficial similarity, and our stimuli were more typical of the "structural-only" similarity found in source-target conditions that fail to find spontaneous analogical transfer than typical of the "superficial" similarity conditions that find spontaneous analogical transfer.

\section{Procedure}

Participants read instructions with a cover story about being the editor of Puzzle Magazine to prevent participants from expecting a relationship between source and target problems. They were informed that they would be working on eight puzzles, but that they would have to rate other puzzles for difficulty (on a scale from 1 to 5 ) as well. Their secretary (the experimenter) would from time to time put a puzzle (i.e., either an analogous cue or distracter cue) on their desk, and they should immediately read through it (including the answer on the back), rate it for difficulty, and hand it back. It was stressed that they should work fast, and that they could return to previous puzzles at any time.

The experiment was carried out on one participant at a time. Participants were given $45 \mathrm{~min}$ to solve all eight problems. During this time they could freely move between problems. Progress on the problems was videotaped. Every $5 \mathrm{~min}$ the participants were handed an analogous or distracter rating task. These tasks were handed to the participants at a time when they had already worked on the relevant problem, and moved on (i.e., after reaching a self-generated impasse). Through a computer linked to the video camera, the experimenter kept track of which problems had been worked on and left (i.e., where the participant had reached an impasse on the problem) and which problem the participant was currently working on. This procedure made it possible to constantly update a pool of available tasks, wherefrom a task was randomly selected (using random number tables) and handed to the participant every $5 \mathrm{~min}$. When the $45 \mathrm{~min}$ had elapsed, the participant was asked to fill out questionnaires about memory for the problems and awareness of the rating task/problem relationship. Impasse was coded as the time when a participant left a problem after having first worked on it, without a correct solution. Access was coded based on which page was turned to next in the booklet. A correct access was defined as returning to the relevant previously unsolved problem as the very next problem following the analogous rating task. Mapping was coded by performance (i.e., correct or incorrect solution). Time spent working on individual problems, both before and after reaching an impasse, was also measured. See Figure 2 for an example of typical problem solving behavior.

\section{Results}

\section{Successful Analogical Mapping}

Participants solved $44 \%$ of the problems. Of the solved problems, $39 \%$ were solved prior to impasse and the remaining $61 \%$ were solved after returning to the problem. Problems solved prior to impasse were excluded from further analysis. A resolution score (e.g., Smith \& Blankenship, 1991-see Appendix B) was calculated for each participant for the analogous-cued problems and the distracter-cued problems, respectively. The resolution score calculates the proportion of impassed problems that were eventually solved. Mean per participant resolution scores were .53 and .17 for the analogous-cued and distracter-cued problems, respectively (standard errors were .05 and .04 , respectively). Analogous cues produced noticeably higher resolution scores, $t(39)=5.62, p<.001$, showing that the cues were highly effective and produced a large effect over a control of the distracter-cued problems that were initially unsolved and left. It could be argued that this comparison does not directly measure a traditional incubation effect as such because no control group working continuously was included; perhaps the distracter-cued problems 


\begin{tabular}{|c|c|c|c|c|c|}
\hline \multicolumn{2}{|c|}{ Time } & Subject behavior & $\begin{array}{l}\text { Provides } \\
\text { solution? }\end{array}$ & Correct? & $\begin{array}{l}\text { Immediate } \\
\text { retrieval? }\end{array}$ \\
\hline $0: 00$ & & Opens booklet and turns page to Problem \#1 & No & & \\
\hline $2: 47$ & & Turns page to Problem \#2 & No & & \\
\hline $4: 39$ & & Turns page to Problem \#3 & Yes & No & \\
\hline & $5: 10$ & Is handed irrelevant cue & & & \\
\hline & $6: 03$ & Hands cue back & & & \\
\hline $6: 54$ & & Turns page to Problem \#4 & No & & \\
\hline $8: 08$ & & Turns page to Problem \#5 & Yes & Yes (excluded) & \\
\hline $8: 36$ & & Turns page to Problem \#6 & Yes & Yes (excluded) & \\
\hline & $10: 12$ & Is handed relevant cue for Problem $\# 3$ & & & No \\
\hline & $10: 41$ & Hands cue back & & & \\
\hline $10: 56$ & & Turns page to Problem \#7 & Yes & No & \\
\hline $14: 48$ & & Turns page to Problem \#8 & Yes & Yes (excluded) & \\
\hline & $15: 12$ & Is handed irrelevant cue & & & \\
\hline & $16: 21$ & Hands cue back & & & \\
\hline & & ALL PROBLEMS SEEN & & & \\
\hline $19: 23$ & & Turns page to Problem \#3 & Yes & Yes & \\
\hline & $20: 15$ & Is handed irrelevant cue & & & \\
\hline & $20: 28$ & Hands cue back & & & \\
\hline $21: 20$ & & Turns page to Problem \#4 & No & & \\
\hline $22: 50$ & & Turns page to Problem \#2 & Yes & Yes & \\
\hline $24: 55$ & & Turns page to Problem \#1 & No & & \\
\hline & $25: 10$ & $\begin{array}{l}\text { Is handed relevant cue for Problem \#5 } \\
\text { (already solved) }\end{array}$ & & & (excluded) \\
\hline & $25: 44$ & Hands cue back & & & \\
\hline $29: 18$ & & Turns page to Problem \#4 & & & \\
\hline & $30: 18$ & Is handed relevant cue for Problem \#1 & & & Yes \\
\hline & $31: 17$ & Hands cue back & & & \\
\hline $31: 18$ & & Turns page to Problem \# 1 & Yes & Yes & \\
\hline $34: 40$ & & Turns page to Problem \#4 & No & & \\
\hline & $35: 21$ & Is handed irrelevant cue & & & \\
\hline & $35: 34$ & Hands cue back & & & \\
\hline & $40: 14$ & Is handed relevant cue for Problem $\# 7$ & & & Yes \\
\hline & $40: 40$ & Hands cue back & & & \\
\hline $40: 50$ & & Turns page to Problem \#7 & No & & \\
\hline $41: 13$ & & Turns page to Problem \#4 & Yes & No & \\
\hline $45: 01$ & & $\begin{array}{l}\text { Time elapsed - experimenter removes } \\
\text { booklet }\end{array}$ & & & \\
\hline
\end{tabular}

Figure 2. Example of problem solving behavior: Participant 35.

would also have an incubation effect (of the autonomous rather than interactive kind) had they been compared to a control group working continuously. What it does demonstrate, however, is that an incubation effect based on new relevant information from the environment is significantly larger than any potential incubation effects based on autonomous cognitive processes alone (.36 vs. .17 at most). Our apparent interactive in- cubation effect could still potentially be explained away if the participants spent more time on the analogous-cued problems after returning to the problems. Bowden (1985) had similar objections to the original Gick and Holyoak (1980) study. To test for this possibility, we measured how much time participants spent working on distracter-cued and analogous-cued problems after having first reached an impasse on the prob- 


\section{B. T. Christensen and C. D. Schunn}

lem (i.e., after leaving the problem when having first worked on it). Participants spent almost $40 \%$ longer (not shorter) after impasse on distracter-cued problems than analogous-cued problems, $t(39)=2.17, p<.04$, thus ruling out the possibility that participants had simply worked longer on the analogous-cued problems after impasse.

\section{Analogical Access}

Immediate analogical access was measured by examining (using video) which problem the participant would turn to in the booklet as the next problem following the presentation of an analogous cue. On average, participants returned immediately $50 \%$ of the time to the relevant problem. It could be argued that this $50 \%$ should be compared to a theoretical one-seventh chance of going to any particular problem next. However, we used a more accurate baseline based on typical page-turning behavior following the distracter tasks. Two strategies were identified in participants' page turning. Before the participants had seen all problems, a dominant strategy was simply to turn to the next unseen problem in the booklet. This strategy occurred $96 \%$ of the time. The time until participants had seen all problems in the booklet and moved onto other problems was on average $21 \mathrm{~min}$ and $31 \mathrm{sec}$. After the participants had seen all the problems, they appeared to be employing a strategy of locating a previously unanswered problem (i.e., a problem where they had not yet indicated any solution in the answer section of the page) to work on. Following any given distracter task after seeing all problems, participants would on average have $33 \%$ unanswered problems in the booklet-but they would return to these $68 \%$ of the time. This information was used to test whether the participants were returning to the relevant previously un- solved problems significantly more often than their usual behavior on the distracter tasks would predict.

For each participant, an immediate access rate and baseline was calculated (see Appendix B) BEFORE and AFTER all problems had been seen (see Table 1). In both cases, access rates were significantly above baseline.

This immediate access measure does not take into account that the participants may become informed (i.e., catch onto the relevance of the analogous cues during problem solving). Being informed should be taken into account when trying to estimate spontaneous immediate access. Immediate access rates were higher after seeing all problems than before, $t(23)=$ $3.49, p<.003$, which could suggest that the initial dominant strategy of turning to the next unseen problem depressed the access rates. But it may also indicate that participants caught on during problem solving. To examine this issue further, access rates were divided into a $2 \times 2$ depending on whether participants were expected to have become informed, and whether they had seen all problems (see Table 2). Participants were scored as "informed" if they had returned immediately to a relevant problem at least once before. Problems that were solved before impasse were once again excluded from this analysis. A small number of problems where the participant had not yet immediately accessed a relevant problem, but where it was indeterminable whether the participants had caught on, because they had received analogous cues to problems they had already solved, were excluded from this analysis.

Comparing rows in Table 3 illustrates that there is an effect of being informed, $\chi^{2}(1)=16.64, p<.001$. Comparing columns in Table 3 shows that there is an effect of page-turning strategy (lower retrieval rates prior to seeing all problems), $\chi^{2}(1)=13.62, p<.001$.

Table 1. Mean Immediate Access Rates and Baseline Calculations Before and After Seeing All Problems

\begin{tabular}{lcccc}
\hline & $\boldsymbol{N}$ & $\boldsymbol{M}$ & $\boldsymbol{S E}$ & $\boldsymbol{t}$ \\
\hline Access rate BEFORE seeing all problems & 25 & .26 & .08 & 3.04 \\
Baseline BEFORE seeing all problems & 25 & .01 & .00 & .007 \\
Access rate AFTER seeing all problems & 39 & .64 & .06 & 7.56 \\
Baseline AFTER seeing all problems & 39 & .17 & .01 & $<.001$ \\
\hline
\end{tabular}


Table 2. Access Rates by Being Informed and Page-Turning Strategy (Whether Participant Had Seen All Problems in the Booklet)

\begin{tabular}{llc}
\hline & Not Seen All & Seen All \\
\hline Not caught on & $.24(n=34)$ & $.46(n=41)$ \\
Caught on & $.60(n=5)$ & $.78(n=36)$ \\
\hline
\end{tabular}

Note. In the figure, $n$ refers to number of problems in each category across participants. Excluded are problems where it was indeterminable whether the participant had caught on and problems solved preimpasse.

To estimate spontaneous immediate retrieval, we excluded all problems where the participant was expected to have caught on, and calculated a mean access rate and baseline per participant following the same procedure as for immediate access measures. The results were still highly significant for both before, $t(24)$ $=3.10, p<.006$, and after, $t(23)=5.78, p<.001$, seeing all problems in the booklet. Means before seeing all problems were .28 and .01 for the baseline; means after seeing all problems were .66 and .16 for the baseline. Note that these means are within-subject, and therefore differ from the means presented in Table 3, which are across-subjects. These results indicate that when spontaneous immediate access to analogous unsolved problems is measured against a control of the same person's problem-solving behavior following distracter tasks, a large effect is found when controlling for participants who become informed during the experiment.

The results show that, when participants were given relevant cues, they deviated from their typical page-turning strategies to return to the relevant problem to try to utilize the new information to solve that problem. They did not randomly look through the booklet to try to find a match for the cue, but rather they went straight to the relevant problem, suggesting that

Table 3. Mean per Category Resolution Scores by Whether Participant Did or Did Not Immediately Return to the Relevant Problem on Seeing an Analogous Cue

\begin{tabular}{lccc}
\hline & \multicolumn{2}{c}{ Analogous Cue } & \\
\cline { 2 - 3 } & $\begin{array}{c}\text { Immediate } \\
\text { Return }\end{array}$ & $\begin{array}{c}\text { Nonimmediate } \\
\text { Return }\end{array}$ & Distractor Task \\
\hline $\begin{array}{c}\text { Resolution } \\
\text { score }\end{array}$ & $.67(n=64)$ & $.32(n=62)$ & $.16(n=135)$ \\
\hline
\end{tabular}

Note. $n$ refers to number of problems in each category. All means differed significantly pairwise $(.001<p<.012)$. becoming informed did not change behavior in the absence of access. Using the same participants' typical page-turning behavior following distracter tasks as a control measure, and controlling for participants who become informed, thus revealed an immediate access effect. It was also found, as it has been many times in the literature, that the immediate access rate was larger for informed participants than for uninformed participants.

\section{What Factors Predict Immediate Retrieval?}

Analogous cues did not lead to $100 \%$ immediate retrieval. Examining what factors predicted immediate retrievals can provide further information on the mechanisms of analogical retrieval in insight problems. From a simple memory perspective, one would predict that encoding time on the impassed problems would predict how easily they are retrieved on cuing. To test whether encoding time predicted immediate access, we measured time spent working on each problem for each participant (i.e., how much time was spent working on each individual page in the booklet) prior to receiving the analogous cue. Then we conducted a within-subject $t$ test comparing encoding time for problems that were immediately accessed, with those that were not. The result was significant, $t(26)=3.81, p$ $<.002$, showing that encoding time did predict immediate retrieval. Means for immediate access and non-immediate access were $3 \mathrm{~min} 49 \mathrm{sec}$ and $2 \min 12$ sec, respectively.

From the perspective of autonomous incubation effects, time away from the impassed problem might predict degree of immediate access. We tested whether time away from the problem (incubation time) predicted immediate access, by measuring the time from last leaving the problem to presentation of the analogous cue. Similar to the encoding time analysis, we then compared incubation time for problems that were immediately accessed, with those that were not. This comparison was not significant, $t(26)=-.57, p>.57$. Means for immediate access and nonimmediate access were $5 \mathrm{~min} 49 \mathrm{sec}$ and $6 \mathrm{~min} 35 \mathrm{sec}$, respectively.

\section{Is Immediate Analogical Mapping Different From Later Returns?}

Because both access and mapping were measured separately in the same design, it was possible to calcu- 


\section{B. T. Christensen and C. D. Schunn}

late whether resolution scores for the immediate and nonimmediate returns differed, and differed from resolution scores for the problems receiving only distracter tasks (see Table 3).

For problems where the participants accessed the relevant problem immediately there was a higher resolution score than for either the nonimmediate returns or the problems receiving only distracter tasks. But on problems where the participant did not immediately access the relevant problem, they still had a significant effect of relevant cuing on performance compared to the problems receiving distracter task. This result could potentially be accounted for if the nonimmediate returns benefitted performance by a process similar to semantic priming (see, e.g., Schunn \& Dunbar, 1996, for another analogical transfer study finding a priming effect). In semantic priming participants are unaware that their performance is increased by relevant information. Because we measured awareness of each problem-cue relationship after the experiment, we were able to test for this possibility. In the nonimmediate return category participants reported being aware of the relationship between the problem and the cue for 17 of the 20 (i.e., 85\%) problems that were solved. This result gives a clear indication that once mapping took place, participants were explicitly aware of the problem-cue relationship. So semantic priming without explicit awareness appeared not to be the cause.

\section{Discussion}

The novel design developed in this experiment showed it was possible to separate a number of constructs for each problem in a within-subject design using video. These constructs, which are typically only manipulated across experimental conditions, include encoding time, impasse, incubation time, analogical access, and analogical transfer, along with measures of becoming informed and problem solving strategies. The careful time-course analysis used here thus has the potential for informing a number of theories in the analogical problem solving and incubation-insight literature

This experiment was able to show that analogical cuing led to immediate access, when controlling for becoming informed, by using the participants' typical problem-solving behavior following distracter problems as a control.

The results suggest that perhaps the failure of much of the previous analogical transfer literature to find such a spontaneous transfer effect may in part be attributable to their use of comparison groups. Previous studies have often showed that, when comparing informed and uninformed participants, the informed participants accessed and transferred more frequently than the uninformed. But perhaps this comparison is unfair when trying to establish whether and how frequently spontaneous immediate access occurs. A large effect of analogous cuing on spontaneous immediate access was thus found when using the same participants' typical problem solving behavior following distracter tasks as a baseline. This experiment also showed, as has often been noted, that when participants became informed (i.e., caught on) they accessed more frequently. Thus, there appears to be a spontaneous immediate access effect of receiving analogous cues, but an even larger effect of being informed. This latter effect of being informed should not, however, lead one to assume that no spontaneous access effect is present even though it may appear small in comparison to the effect of being informed (as, e.g., Anolli et al., 2001, seems to do).

Encoding time predicted immediate access, thus supporting Dunbar's (2001) hypothesis that encoding time of the analogue to be accessed is extremely important, and may help explain why some researchers have failed to find transfer effects. Incubation time, however, did not predict immediate access suggesting that autonomous incubation effects played little role in this study.

The Opportunistic Assimilation (Seifert et al., 1995) theory of incubation was also supported by this spontaneous immediate access effect, and OA was further supported by the result that receiving an analogous problem during incubation (after impasse) increased performance through analogical mapping. These results lend support to the OA theory that-following impasse on creative problems - a chance encounter with analogous relevant new information in the environment will (at least in some cases) spontaneously trigger access and mapping to the previously unsolved problem. Thus, presenting analogous problems during incubation is at least one reliable way of demonstrating incubation effects, unlike visual analogies and associates in RAT problems, which, as noted in the introduc- 
tion, have shown mixed results. On at least some analogical problems, chance does appear to favor the prepared mind.

Several more experiments should be conducted to further test OA theory. For example, the theory would predict that spontaneous immediate access rates should increase after impasse. This prediction could potentially be tested by presenting cues after impasse (as it is done in this experiment) and comparing them with other problems where the cue is presented before impasse (possibly before seeing the target as it is done in standard analogical problem solving). This design is essentially the same variation that was done in the original Gick and Holyoak (1980, exp V) study. They found no significant difference, but this result should be retested using a variation of this design in a larger study.

From the retrospective reports, participants were in most cases explicitly aware of the problem-cue relationship. This result means that a limitation of this study is that it cannot purport to explain incubation phenomena where participants are unaware of how they solved the problem. Much anecdotal evidence concerns individuals who later reported that an idea just simply occurred to them when they for example were in the bath or sitting on a bus. Unless a mechanism can be identified that would explain why people later often do not report that they used cues from the environment in their solution of the problem, OA may not be able to explain these cases. However, it could be accounted for by the standard memory mechanism of differential rehearsal or retrieval practice (e.g., Bjork, 1988). Perhaps when a creative discovery or invention is rehearsed over the years through repeated retelling of the problem and solution by its creator, memory of how the problem was originally solved is not rehearsed as frequently. In this case, standard forgetting processes in addition to OA may account for the phenomenon.

Finally, this experiment may seem to lack ecological validity and may appear somewhat contrived and artificial. As other studies on analogy have shown (e.g., Dunbar, 2001; Blanchette \& Dunbar, 2001), this difference between experimental settings and real-world context is sometimes very important and may lead to different results. Therefore it remains to be seen whether more naturalistic research will be able to find evidence of these spontaneous access and incubation effects. We are presently collecting data on design problem solving using Dunbar's $(1995,1997)$ in vivo methodology to answer this question.

\section{References}

Adams, L. T., Kasserman, J. E., Yearwood, A. A., \& Perfetto, G. A. (1988). Memory access: The effects of fact-oriented versus problem-oriented acquisition. Memory and Cognition, 16, 167-175.

Anolli, L., Antonietti, A., Crisafulli, L., \& Cantoia, M. (2001). Accessing source information in analogical problem-solving. Quarterly Journal of Experimental Psychology: Human Experimental Psychology, 54A, 237-261.

Bjork, R. A. (1988). Retrieval practice and the maintenance of knowledge. In M. M. Gruneberg, P. E. Morris, \& R. N. Sykes (Eds.), Practical aspects of memory: Current research and issues (pp. 396-401). Chichester, UK: Wiley.

Blanchette, I., \& Dunbar, K. (2001). Analogy use in naturalistic settings: The influence of audience, emotion, and goals. Memory and Cognition, 29, 730-735.

Bowden, E. M. (1985). Accessing relevant information during problem solving: Time constraints on search in the problem space. Memory \& Cognition, 13, 280-286.

Browne, B. A., \& Cruse, D. F. (1988). The incubation effect: Illusion or illumination? Human Performance, 1, 177-185.

Campbell, D. T. (1960). Blind variation and selective retentions in creative thought as in other knowledge processes. Psychological Review, 67, 380-400.

Chen, Z. (1995). Analogical transfer: From schematic pictures to problem solving. Memory and Cognition, 23, 255-269.

Clement, C. A., Mawby, R., \& Giles, D. E. (1994). The effects of manifest relational similarity on analog retrieval. Journal of Memory and Language, 33, 396-420.

Dodds, R. A., Smith, S. M., \& Ward, T. B. (2002). The use of environmental clues during incubation. Creativity Research Journal, 14, 287-304.

Dodds, R. A., Ward, T. B., \& Smith, S. M. (in press). A review of the experimental literature on incubation in problem solving and creativity. In M. A. Runco (Ed.), Creativity research handbook (3rd ed.). Cresskill, NJ: Hampton.

Dominowski, R. L. \& Dallob, P. (1995). Insight and problem solving. In R. J. Sternberg \& J. E. Davidson (Eds.), The nature of insight (pp. 33-62). Cambridge, MA: MIT Press.

Dominowski, R. L., \& Jenrick, R. (1972). Effects of hints and interpolated activity on solution of an insight problem. Psychonomic Science, Mar-338.

Dorfman, J. (1990). Metacognitions and incubation effects in insight problem solving. Unpublished dissertation for the degree of Doctor of Philosophy University of California, San Diego.

Dorfman, J., Shames, V. A., \& Kihlstrom, J. F. (1996). Intuition, incubation, and insight: Implicit cognition in problem solving. In G. D. M. Underwood (Ed.), Implicit cognition (pp. 257-296). New York: Oxford University Press. 


\section{B. T. Christensen and C. D. Schunn}

Dreistadt, R. (1969). The use of analogies and incubation in obtaining insights in creative problem solving. Journal of Psychology, $71,159-175$.

Dunbar, K. (1995). How scientists really reason: Scientific reasoning in real-world laboratories. In R. J. Sternberg \& J. E. Davidson (Eds.), The nature of insight (pp. 365-395). Cambridge, MA: MIT Press.

Dunbar, K. (1997). How scientists think: On-line creativity and conceptual change in science. In T. B. Ward, S. M. Smith, \& J. Vaid (Eds.), Creative thought: An investigation of conceptual structures and processes (pp. 461-493). Washington, DC: American Psychological Association.

Dunbar, K. (2001). The analogical paradox: Why analogy is so easy in naturalistic settings yet so difficult in the psychological laboratory. In D. Gentner, K. J. Holyoak, \& B. N. Kokinov (Eds.), The analogical mind: Perspectives from cognitive science. (pp. 313-334). Cambridge, MA: MIT Press.

Duncker, K. (1945). On problem-solving. Westport, CT: Greenwood.

Forbus, K. D., Gentner, D., \& Law, K. (1994). MAC/FAC: A model of similarity-based retrieval. Cognitive Science, 19, 141-205.

Gentner, D., Rattermann, M. J., \& Forbus, K. D. (1993). The roles of similarity in transfer: Separating retrievability from inferential soundness. Cognitive Psychology, 25, 524-575.

Gick, M. L., \& Holyoak, K. J. (1980). Analogical problem solving. Cognitive Psychology, 12, 306-355.

Gick, M. L., \& Holyoak, K. J. (1983). Schema induction and analogical transfer. Cognitive Psychology, 15, 1-38.

Hammond, K. J., Seifert, C. M., \& Gray, K. C. (1991). Functionality in analogical transfer: A hard match is good to find. Journal of the Learning Sciences, 1, 111-152.

Holyoak, K. J., \& Koh, K. (1987). Surface and structural similarity in analogical transfer. Memory \& Cognition, 15, 332-340.

Hummel, J. E., \& Holyoak, K. J. (1997). Distributed representations of structure: A theory of analogical access and mapping. Psychological Review, 104, 427-466.

Kaplan, C. A., \& Simon, H. A. (1990). In search of insight. Cognitive Psychology, 22, 374-419.

Keane, M. (1985). On drawing analogies when solving problems: A theory and test of solution generation in an analogical problem-solving task. British Journal of Psychology, 76, 449-458.

Lockhart, R. S., Lamon, M., \& Gick, M. L. (1988). Conceptual transfer in simple insight problems. Memory and Cognition, 16, $36-44$.

Mednick, M. T., Mednick, S. A., \& Mednick, E. V. (1964). Incubation of creative performance and specific associative priming. Journal of Abnormal and Social Psychology, 69-88.

Mednick, S. A. (1962). The associative basis of the creative process. Psychological Review, 69, 220-232.

Ohlsson, S. (1984). Restructuring revisited: I. Summary and critique of the Gestalt theory of problem solving. Scandinavian Journal of Psychology, 25, 65-78.

Olton, R. M. (1979). Experimental studies of incubation: Searching for the elusive. Journal of Creative Behavior, 13, 9-22.

Olton, R. M., \& Johnson, D. M. (1976). Mechanisms of incubation in creative problem solving. American Journal of Psychology, 89, 617-630.
Patalano, A. L., \& Seifert, C. M. (1994). Memory for impasses during problem solving. Memory and Cognition, 22, 234-242.

Patalano, A. L., \& Seifert, C. M. (1997). Opportunistic planning: Being reminded of pending goals. Cognitive Psychology, 34, $1-36$.

Perkins, D. N. (2000). Archimedes' bathtub The art and logic of Breakthrough Thinking. New York: WW Norton.

Posner, M. I. (1973). Cognition: An introduction. Glenview, IL: Scott.

Ross, B. H. (1987). This is like that: The use of earlier problems and the separation of similarity effects. Journal of Experimental Psychology: Learning, Memory, \& Cognition, 13, 629-639.

Ross, B. H. (1989). Distinguishing types of superficial similarities: Different effects on the access and use of earlier problems. Journal of Experimental Psychology: Learning, Memory, \& Cognition, 15, 456-468.

Ross, B. H., Ryan, W. J., \& Tenpenny, P. L. (1989). The access of relevant information for solving problems. Memory \& Cognition, 17, 639-651.

Schunn, C. D., \& Dunbar, K. (1996). Priming, Analogy, \& Awareness in complex reasoning. Memory and Cognition, 24, 271-284.

Seifert, C. (1994). The role of goals in retrieving analogical cases. In J. A. Barnden \& K. J. Holyoak (Eds.), Analogy, metaphor, and reminding (pp. 95-168). Norwood, NJ: Ablex.

Seifert, C. M., Meyer, D. E., Davidson, N., Patalano, A. L., \& Yaniv, I. (1995). Demystification of cognitive insight: Opportunistic assimilation and the prepared-mind perspective. In R. J. Sternberg \& J. E. Davidson (Eds.), The nature of insight (pp. 65-124). Cambridge, MA: MIT Press.

Silveira, J. M. (1971). Incubation: The effect of interruption timing and length on problem solution and quality of problem processing. Unpublished doctoral dissertation University of Oregon.

Simon, H. A. (1966). Scientific discovery and the psychology of problem solving. In R. G. Colodny (Ed.), Mind and cosmos (pp. 22-41). Pittsburgh, PA: University of Pittsburgh Press.

Smith, S. M. (1995). Fixation, incubation, and insight in memory and creative thinking. In S. M. Smith, T. B. Ward, \& R. A. Finke (Eds.), The creative cognition approach (pp. 135-156). Cambridge, MA: MIT Press.

Smith, S. M., \& Blankenship, S. E. (1991). Incubation and the persistence of fixation in problem solving. American Journal of Psychology, 104, 61-87.

Thagard, P., Holyoak, K. J., Nelson, G., \& Gochfeld, D. (1990). Analogical retrieval by constraint satisfaction. Artificial Intelligence, 46, 259-310.

Zeigarnik, B. (1927). Das Behalten von erledigten und unerledigten Handlungen. Psychologie Forschung, 9, 1-85.

\section{Appendix A: Problems Used}

The insight problems used included slightly changed versions of the elephant problem (Chen, 1995), the 10 trees problem (Dreistadt, 1969), the ten- 
nis tournament problem (Ohlsson, 1984), the sports car racers, 10 matches, shot, and fly problems (all Perkins, 2000), the bracelet problem (Silveira, 1971), as well as other analogical insight puzzles that were generated with elements taken from puzzle books and puzzle sites on the internet. Two detailed examples of a problem and cue are presented below.

\section{PROBLEM: TENNIS TOURNAMENT}

A singles tennis tournament is being planned that will involve 88 players. The tournament will be single elimination, so any player who loses a match is out of the tournament and the winner will be the person who doesn't lose at all. The organizers of the tournament need to know how many matches will be played so they can budget for court time and balls. How many matches will be played in all during the tournament?

\section{CUE: THE VIKINGS}

The Vikings believed that by dying on the battlefield they would be allowed a place in Viking heaven (Valhalla). Forty-four Vikings are assembled to battle each other until only one is left standing. However, even Vikings have rules; the Vikings fight one-on-one at a time, until one of them is dead. Once the battle begins all Vikings will start fighting with the nearest other Viking. The Viking who is still alive then moves on to find a new Viking to fight, while the loser goes to Valhalla. And so on. What is the number of one-on-one Viking fights there will be in this battle?

Answer: The number of one-on-one Viking fights will necessarily equal the number of dead Vikings (i.e., Vikings that go to Valhalla). Therefore there will be 43 one-on-one Viking fights before the battle is over and only one is left standing.

\section{PROBLEM: MARY AND ELIZA}

Mary and Eliza were ardent rivals in sports car racing, socializing, and the game of life in general. Mary's husband got sick of their competitiveness and decided to teach them a lesson. He asked them to meet him at a deserted racetrack one day with their sports cars. He announced, "The winner of the race will get a brand new sports car. But this is a race with a difference. The one whose car crosses the finish line last wins. Marry and Eliza hopped into the cars and roared off around the track as fast as they could go. Why?

\section{CUE: THE ESTATE}

On his deathbed, a rancher came up with a scheme to pass on his estate. He called in his two sons, and told them to each take their horse and race to the nearest town and back. The horse that came second would win the estate for its owner. The young cowboys set out but were obviously trying to go as slow as possible to finish second. They were stopped by a knowledgeable villager and asked what they were doing. The sons explained the situation to the villager. The villager thought for a minute then came up with an idea that had the sons hurtling off at breakneck speed. What did the villager propose?

Answer: "Take the other's horse"

\section{Appendix B: Calculation Methods}

\section{Calculation Methods for Resolution Scores}

Resolution scores by participant were calculated by applying the following equation:

$$
\frac{X_{i}-Y_{i}}{4-Y_{i}}
$$

In the equation, $X$ refers to number of problems solved ultimately, $Y$ refers to number of problems solved before impasse (i.e., before leaving problem the first time), and $i$ refers to $i$ th participant. This was done for both the analogous cues, and the distracter cues. 


\section{B. T. Christensen and C. D. Schunn}

Calculation Methods for Immediate Access and Spontaneous Immediate Access Rates, Before and After Seeing All Problems

Access rates were calculated by applying the following equations:

$$
\frac{\sum_{j=1}^{p} X_{i j}}{p_{i}}
$$

In the equation, $X$ refers to whether participant did (1) or did not (0) immediately turn to the relevant page in the booklet following cue; $p$ refers to number of analogous-cued problems that were unsolved prior to cuing; $i$ refers to $i$ th participant, and $j$ to $j$ th cued problem. The problem sample size of $p_{i}$ varied depending on condition: before/after seeing all problems in the booklet; and before/after catching on.

\section{Calculation Methods for the Baseline for Immediate Access and Spontaneous Immediate Access Rates, Before and After Seeing All Problems}

Baselines for access rates were calculated by examining typical problem-solving behavior following the distracter cues. Following distracter cues and before seeing all problems, participants would simply turn to the next unseen problem $96 \%$ of the time. After seeing all problems, participants would return to previously unanswered problems $68 \%$ of the time, and to answered problems $32 \%$ of the time. Baselines were thus calculated by applying the equations:

Baseline before seeing all problems:

$$
\frac{\sum_{j=1}^{p}\left(\frac{0.04}{k_{i j}}\right)}{p_{i}}
$$

Baseline after seeing all problems:

$$
\frac{\sum_{j=1}^{p}\left(\frac{m_{i j}}{.68 u_{i j}+.32\left(7-u_{i j}\right)}\right)}{p_{i}}
$$

In the equations, $k$ refers to number of seen problems at cue time; $u$ to number of unanswered problems at cue time (excluding the problem the participant is currently working on), the value of $m$ is .32 if the cued problem has already been answered whereas it is .68 if it has not, $p$ refers to number of distracter-cued problems that were unsolved prior to cuing, $i$ refers to $i$ th participant, and $j$ to $j$ th cued problem. The problem sample size of $p_{i}$ varied depending on condition: before/after seeing all problems in the booklet; and before/after catching on). 